\title{
LOS EDIFICIOS DE LAS BIBLIOTECAS PÚBLICAS EN LOS PROGRAMAS ELECTORALES (CASTILLA-LA MANCHA, 2007-2015)
}

\author{
José Pablo Gallo León* \\ Universidad de Alicante \\ María Dolores Marín Jiménez ** \\ Biblioteca Municipal de Hellín, Albacete. \\ José-Antonio Gómez-Hernández,** \\ Facultad de Comunicación y Documentación. Universidad de Murcia.
}

\begin{abstract}
Resumen: Se analizan las propuestas sobre edificios e instalaciones de las bibliotecas presentes en los programas con que los partidos políticos participaron en las elecciones de Castilla-La Mancha durante el periodo de la crisis económica, con el fin de evaluar cómo reflejan el interés social por las mismas. A pesar de la desmaterialización digital de los servicios bibliotecarios en el actual entorno híbrido, la proyección de la biblioteca pública como un servicio a la sociedad requiere de una presencialidad física que dé soporte a su actual papel como ágora, punto de intercambio socio-intelectual y de formación de la comunidad a la que sirve. Esto debería verse traducido en un interés social por los espacios bibliotecarios que puede ser testado a través de su presencia en los programas electorales, donde se ven reflejados los temas que conforman la agenda política. Concluimos que ha habido un cambio de tendencia, priorizando la reforma y aseguramiento de la apertura de los centros frente a la construcción de nuevas bibliotecas. Igualmente, se comprueba que los programas presentan una visión muy tradicional de los servicios presenciales.
\end{abstract}

Palabras clave: Bibliotecas públicas; edificios de biblioteca; biblioteca como lugar; políticas públicas; programas electorales; política bibliotecaria; políticas culturales; elecciones autonómicas; elecciones locales; Castilla-La Mancha.

Title: PUBLIC LIBRARIES BUILDINGS AND FACILITIES IN THE ELECTORAL PROGRAMS (CASTILLALA MANCHA, SPAIN. 2007-2015).

Abstract: This paper presents an analysis of proposals about library building and facilities identified in the electoral programs published by the Political Parties competing in regional elections during the economic crisis in Castilla-La Mancha. The objective was to evaluate how these proposals reflect social and political interest in public libraries. Despite the digital dematerialization of library services in nowadays hybrid environment, the projection of the public library as a service to society requires a physical presentiality that supports its current role as an agora, a place for socio-intellectual exchange and training for its community. This should be translated into a social interest in library spaces that can be tested through its presence in the electoral programs, which reflect the issues that shape the political agenda. The work concludes a trend that prioritizes the reform and continuity of the libraries rather than projects for new libraries. It is also found that the programs have a very traditional view of on-site services.

Keywords: Public libraries; library buildings; library as place; public policy; electoral programmes; cultural policy; regional elections; local elections; Castilla-La Mancha.

\section{INTRODUCCIÓN}

En este trabajo analizamos el interés de los partidos políticos por la biblioteca y concretamente por sus instalaciones, edificios o infraestructuras, identificando las propuestas que recogen en sus programas en los procesos electorales. Tras encuadrar nuestra investigación en los estudios relativos a las relaciones entre política y bibliotecas y presentar una visión del sentido de los espacios bibliotecarios en una sociedad de información digital, estudiamos los procesos electorales autonómicos y locales de 2007 y 2015 en Castilla-La Mancha para valorar en qué medida los partidos políticos conocen y valoran la función de los espacios bibliotecarios tal y como se concibe actualmente desde la Biblioteconomía.

Los edificios de biblioteca llevan siendo puestos en duda casi medio siglo. Por una parte, el recorrido hacia la biblioteca digital parecía destinar el espacio material de la misma a su desaparición. Por otra, este mismo avance de las

\footnotetext{
*jpablogallo@ua.es

***lola.marin@hellin.es

*** jgomez@um.es
}

Recibido: 02-10-2016; 2 $2^{\mathrm{a}}$ versión: 25-01-2017; aceptado: 08-02-2017.

GALLO LEÓN, J.P.; MARÍN JIMÉNEZ, M.D. y GÓMEZ HERNÁNDEZ, J.A. Los edificios de las bibliotecas públicas en los programas electorales (Castilla-La Mancha, 2007-2015). Anales de Documentación, 2017, vol. 20, $\mathrm{n}^{\circ}$ 1. Disponible en: http://dx.doi.org/10.6018/analesdoc.20.1.26995. 
tecnologías hacia la sociedad red lleva a poner en duda la propia existencia de la biblioteca, sea cual sea su entorno de servicio. El propio nombre de la biblioteca nos retrotrae a su condición física como espacio y colección documental, generando una incertidumbre que se refleja en múltiples estudios profesionales que realizan prospectiva del futuro de las bibliotecas. Además, la biblioteca puede pasar a tener una nueva naturaleza líquida (Gil Solés, 2012), para así poder fluir y llegar donde se la necesita, rompiendo las barreras del espacio físico convencional.

Sin embargo, a pesar de la desmaterialización digital de los servicios bibliotecarios en el actual entorno híbrido, la proyección de la biblioteca pública como un servicio a la sociedad requiere de una presencialidad física, especialmente para el cumplimiento de su papel como ágora o tercer lugar, punto de intercambio socio-intelectual de la comunidad a la que sirve. Ésta es una de las principales proyecciones de futuro de la biblioteca, refrendada por estudios de prospectiva que se citan más adelante.

Esto se debería ver reflejado en un interés social en que los espacios bibliotecarios alcancen un nivel adecuado a las necesidades del servicio. Como se sabe, este interés compartido es determinante para la incorporación de un asunto a la agenda política y, desde ese momento, para ser objeto de atención de la acción de gobierno. Una forma de comprobar su presencia actual es el estudio de los programas electorales, donde se ven reflejados los temas que conforman la citada agenda.

Por otra parte, la crisis económica ha afectado especialmente a la construcción y reforma de espacios bibliotecarios. Al respecto, el ejemplo de Castilla-La Mancha resulta paradigmático dentro del panorama nacional, por lo que supuso un truncamiento de una situación de gran desarrollo de los sistemas de lectura pública en los años 90 y 2000. Se partió de una realidad particularmente pobre para mejorar de forma notable gracias al impulso de políticas bibliotecarias de gran calado y que resultaron ejemplares. Así, resulta especialmente adecuado tomar esta región como objeto de estudio, pues nos proporciona resultados representativos de lo que ha podido pasar en otras regiones españolas o europeas.

Igualmente, resulta significativo establecer un análisis comparativo entre dos elecciones, las de 2007 y 2015 , puesto que en la primera los programas aún se realizaron en un ámbito presupuestario expansivo, mientras que en las segundas ya se han vivido los peores años de la crisis y el hundimiento de los presupuestos de servicios del ámbito de la cultura o la educación.

A pesar de la relevancia que el análisis de las políticas bibliotecarias puede tener para estas instituciones y sus gestores, es escasa la bibliografía que trata de cuestiones como la visibilidad electoral de las bibliotecas. Y tampoco se ha analizado, tras la reciente crisis económica, si se ha producido como consecuencia un cambio de modelo, en el que los edificios e infraestructuras hayan dejado de ser objeto de interés prioritario dentro de las políticas bibliotecarias. Reflexionar sobre todas estas cuestiones es la motivación del presente trabajo.

\section{OBJETIVOS Y METODOLOGÍA}

Se pretende determinar el grado de interés político en la construcción y mantenimiento de infraestructuras bibliotecarias y su consiguiente incorporación a la agenda política, intentando establecer la evolución durante la crisis económica respecto al interés por otros servicios bibliotecarios. Esta presencia en la agenda política debería reflejar el interés social sobre el tema.

Para ello, hacemos un análisis cuantitativo y cualitativo de la información recopilada de los distintos partidos y propuestas políticas en dos elecciones municipales y autonómicas, las de 27 de mayo de 2007 y las de 24 de mayo de 2015, celebradas en momentos radicalmente distintos desde el punto de vista de las políticas de gasto público. Las primeras reflejarán una mayor disponibilidad económica mientras que las últimas elecciones locales y regionales permitirán observar cómo el largo periodo de crisis ha afectado a los temas de interés para la sociedad y los partidos políticos. Identificamos las propuestas sobre la biblioteca desde varios puntos de vista, las clasificamos temáticamente y valoramos cuestiones de eficacia y eficiencia, adaptación de las propuestas a cada municipio, calidad y posible satisfacción de los ciudadanos.

Intentamos recoger todos los programas de los partidos en los dos procesos electorales objeto de estudio, centrándonos inicialmente en los municipios con más de 1.000 habitantes, porque son los que actualmente tienen obligación de tener biblioteca pública municipal según la Ley 3/2011, de 24 de febrero, de la Lectura y las Bibliotecas de Castilla-La Mancha. Sin embargo, como 625 de los 919 municipios de esta Región tienen una población menor de 
1.000 habitantes $(68 \%)$ y muchos de ellos disponen también de servicio bibliotecario, se han tenido en cuenta igualmente los programas electorales recogidos en esos municipios.

Los programas electorales se recopilaron durante los dos periodos de quince días de campaña electoral, y se completó hasta el mes de julio siguiente a ambos procesos electorales. Hacerlo así fue fundamental para obtener una información completa por el carácter perecedero de estos programas, que los partidos no conservan después de las convocatorias electorales, ni hacen objeto de Depósito Legal, no habiendo otra normativa que obligue a su conservación. Incluso la versión electrónica de los programas presente en las páginas web de los partidos suele desaparecer una vez finalizado el proceso electoral, de modo que es imprescindible recoger los programas en el momento. Ello lo hicimos tanto mediante petición directa a los partidos en sus sedes municipales o regionales, como revisando sus webs y redes sociales, y gracias también a la ayuda de los propios bibliotecarios de las localidades, que fueron un canal importante para la recogida de programas.

Tras el proceso de búsqueda descrito se obtuvieron 428 programas de 314 municipios en 2007; y 389 de 193 en 2015. Una vez recopilados y analizados todos para identificar las propuestas relacionadas con bibliotecas, se extrajeron 339 en total en 2007 y 377 en 2015. La exhaustividad en el proceso de obtención de los documentos para el análisis y la amplitud de esta muestra nos hace considerarla claramente representativa para realizar estimaciones sobre la presencia de las bibliotecas en la agenda política.

Para completar la metodología y poder valorar el grado de cumplimiento de las propuestas electorales, se intentaron cotejar los datos de los edificios e instalaciones realmente construidas e inauguradas en Castilla-La Mancha durante la legislatura 2007-2011 con las propuestas recogidas en los programas de esa primera etapa electoral analizada.

\section{MARCO TEÓRICO}

A pesar del interés que para el bibliotecario debe tener el conocimiento de la política y la determinación de sus prioridades como factor del propio desarrollo y supervivencia de su institución, la bibliografía que vincula ambos mundos es escasa: "lo político [es] un campo, por cierto, comúnmente ajeno a la reflexión bibliotecológica pero definitivo para las prácticas bibliotecarias, especialmente para las de la biblioteca pública” (Jaramillo, Álvarez Zapata, y Moncada, 2005).

Estudiosos del tema como Jaeger, Bertot y Gorham (2013) incluso nos recuerdan la falta de investigación sobre el tema citando un trabajo de David Shavit (1986) en el que consideraba la Public Library Inquiry y la obra de Oliver Garceau (1949) como los últimos grandes trabajos sobre bibliotecas y política hasta ese momento.

Hay algunas revisiones previas desde el ámbito académico y profesional, como las de Meneses Tello (2013), Evjen (2015) y Marín Jiménez (2015) en España; además, el Bulletin des Biliothèques de France consagró uno de sus dosieres (Desrichard, 2011) a la política y las bibliotecas, y hay una revista íntegramente consagrada a aspectos más o menos vinculados con la cuestión de la política bibliotecaria: Progressive Librarian. Algunos trabajos de autores destacados en el tema como Audunson, Usherwood o, en el ámbito hispano, Javier Echeverría, no los consideramos aquí porque su contenido se aleja de los objetivos específicos perseguidos en este artículo.

En España Juan Sánchez $(1994,2015)$ se ha esforzado en sensibilizar sobre el tema escribiendo sobre todo artículos de opinión en la prensa diaria, recopilados posteriormente en libros. Este bibliotecario castellano-manchego ha sido de los pocos que se ha preocupado por denunciar la gravedad de la ausencia de las bibliotecas en los programas electorales, que sería consecuencia de un amplio desconocimiento de los políticos, incluso donde se han llevado políticas activas como en Castilla-La Mancha.

\subsection{Elecciones, agenda política y bibliotecas}

Para conocer de forma empírica, no meramente intuitiva, la presencia de las bibliotecas en la agenda política, se pueden usar, básicamente tres vías:

- Métodos directos, como entrevistas a los políticos y encuestas a la población

- Estudio de las políticas públicas desarrolladas

- Estudio de los programas electorales de los partidos políticos

La agenda política la conforman aquellos temas que son del mayor interés para los políticos y sobre los que se va a actuar realizando diversas iniciativas, tanto legislativas como ejecutivas. Por ejemplo, decidiendo que se va a construir una biblioteca o legislando que las poblaciones de más de un número determinado de habitantes deben contar con servicio permanente de lectura pública. De ahí la importancia del análisis de la agenda y de la implicación del 
bibliotecario en su formación "las bibliotecas públicas no pueden ser olvidadas. Deben estar en la agenda y el programa de cualquier partido político" (Sánchez, 2015).

De esta forma, no podemos obviar que la dotación y propia existencia de la biblioteca depende de una decisión política (Wahnich, 2011), y para ello es condición previa e indispensable que estén presentes en la agenda política.

Según Ballart y Ramió (2000), la agenda es la "lista de temas o problemas a los que los responsables del Gobierno, los parlamentarios, los altos cargos de la Administración y algunas otras personas y grupos estrechamente relacionados con aquellos, prestan atención en cada momento". Por ello, a veces hay diferencias entre lo que interesa a la sociedad y lo que ocupa al Gobierno en cada momento. Siendo la conformación de esta agenda un tema que ha requerido de profundos estudios y diversas teorías, como las de Dahl, Barach y Baratz, Lukes o Kingdon, que no viene al caso citar ni desarrollar. Sólo se debe recordar que en ella interviene un interés social, que debe haber un corpus profesional amplio que ofrezca soluciones y que haya un cambio en la percepción de los políticos para que se abra la llamada "ventana de la oportunidad" (Ballart y Ramió, 2000), en la cual se puede incorporar un tema a la agenda. En ello, los bibliotecarios pueden ejercer influencia y ofrecer las soluciones.

Resulta evidente que los temas que se incorporan a los programas electorales son materias que conforman esta agenda, temas de interés social y que los políticos, al menos en teoría, están dispuestos a abordar y así se lo comunican a sus posibles votantes. Pero, como decíamos, la relación entre la agenda y el interés social no es directa, pues en la conformación de la misma también intervienen otros elementos, como la influencia de lobbistas y la definición del problema, y posibles soluciones para la apertura de la ventana de oportunidad. En todo caso, aceptando que "en los programas se sitúa la conexión entre representantes y representados" (Noguera, 2011), deberían ejemplificar principalmente el interés social. De esta forma, la presencia en los programas electorales no sólo nos muestra lo que se encuentra en la agenda política, sino lo que los políticos creen que interesa a la sociedad.

Sin embargo, se suele entender que el interés político en las bibliotecas es escaso, como concluyen los trabajos de Gazo (2011), Tuleu (2011), Sveum y Tveter (2012) y Michnik (2015), quien lo expresa así: "Los temas relativos a las bibliotecas públicas son políticamente pequeños y no se priorizan en la agenda política". Ante ello, los bibliotecarios nos debemos implicar más, debemos participar e incluir en la conformación de la agenda. Existe un convencimiento profesional sobre el valor social y democrático de las bibliotecas, pero este debe ser transmitido a la propia sociedad y a los políticos. Debemos hacer llegar estas ideas para que no se pierdan en un marasmo de diferentes intereses. Esto es: participar en las discusiones de la denominada por Habermas "esfera pública". Jaeger, Gorham, Bertot y Sarin (2014) insisten en la necesidad de nuestra presencia en ámbitos políticos para incrementar nuestras posibilidades de influencia y asegurar nuestro futuro.

Desgraciadamente, la escasa visibilidad de las bibliotecas en la agenda no deja de evidenciar también, como sabemos por los procesos de conformación de las agendas, una sensibilidad insuficiente de la misma sociedad respecto a nuestras instituciones. En una época de crisis en la que servicios sanitarios y educativos han visto restringidos sus presupuestos, es posiblemente natural que la sociedad dé prioridad a otros servicios, aunque suponga un error a largo plazo. Por ello es importante la acción de los bibliotecarios para dar a conocer a la sociedad la importancia que para su desarrollo tienen las bibliotecas; y para que los políticos conozcan verdaderamente las actuaciones que deben realizar, evitando las divergencias entre demandas sociales, necesidades reales y políticas aplicadas que Boughey y Cooper (2010) detectaron como habituales.

Aún en un contexto negativo como la recesión, y cuando se pone en duda el sentido de los espacios físicos, hay circunstancias que han llevado a la construcción de magníficos edificios de biblioteca, como ha ocurrido en los casos de las ciudades de Aarhus, Birmingham y Oslo. Evjen (2015) estudió las motivaciones por las que se había llegado a la decisión política de hacerlo, en un artículo que por tanto tiene relación directa con nuestros objetivos. Su conclusión es que en estas ciudades la planificación de un nuevo centro no respondió a criterios de necesidad social, educativa y cultural, sino a razones de índole socioeconómico: lograr la revitalización urbanística del entorno o de toda la ciudad, incluso intentando emular el efecto Bilbao con un servicio de mayor proximidad.

\subsection{La permanente necesidad de los espacios de biblioteca}

La vigencia y necesidad de la dimensión física de la biblioteca, la biblioteca de ladrillo y cemento (brick and mortar) como se dice en una acertada imagen en inglés, debe también justificarse. Se trata de una presencialidad física que ya no está supeditada al mero almacenamiento de una colección en papel, que puede ser incluso trasladada parcialmente a depósitos externos y a menudo cooperativos. El espacio que dejan libres esas colecciones sigue siendo de gran valor para la biblioteca, pues ello le permite ofrecer una serie de servicios que antes se veían limitados por la presión ejercida por colecciones en continuo crecimiento. 
La biblioteca física es así necesaria para el cumplimiento del papel que le otorga hoy la sociedad y que señalan estudios sobre el futuro de las bibliotecas (Gallo-León, 2013):

- Biblioteca como ágora, punto de encuentro o tercer lugar. El espacio al que se va por iniciativa propia, al margen del trabajo y el hogar, para socializarse, intercambiar experiencias, pasar el tiempo de ocio.

- Biblioteca como formadora y espacio para el aprendizaje. "El lugar al que siempre puedes dirigirte para aprender algo que necesitas". En ella puedes encontrar la información que requieres para ello, te pueden ayudar a buscarla, te enseñan a hacerlo por tu cuenta o alguien te forma directamente sobre lo que quieres aprender. Además, encuentras el entorno adecuado para el aprendizaje. Y para todo ello no basta con el -necesarioespacio digital, sino que se facilita y enriquece con el espacio físico.

- Biblioteca como espacio de creación. Directamente relacionado con lo anterior, la biblioteca puede proporcionar espacios del tipo maker o medialab, además de puntos de prueba de novedoso aparataje electrónico, para que las personas desarrollen sus proyectos o, más importante, aprendan a hacerlo; bien de forma autónoma, bien guiados.

- Biblioteca social, en la que se dan servicios básicos de ocio, formación, información y conectividad a los sectores de población que no pueden costeárselo, además de proporcionar el marco adecuado para el intercambio y el desarrollo de actividades sociales.

Estos mismos roles, en un orden algo diferente, son defendidos por el reciente estudio para el Cult Committee del Parlamento Europeo (Lison, et al., 2016). En el mismo se insiste en el concepto de tercer lugar y en el de biblioteca como punto de acceso al conocimiento para toda la sociedad. Son espacios sociales, el equipamiento cultural más utilizado (incluso más que los estadios de fútbol) y libres y abiertos a todos, sin connotaciones comerciales. Además, señala su importante papel en el desarrollo urbanístico, siendo reconocido como un interesante vecino por los comercios.

Esta idea, refrendada por realidades conocidas, puede llevarse al paroxismo hablando más de un efecto Seattle que de efecto Bilbao (Worpole, 2013), refiriéndose al ejemplo de la célebre biblioteca pública de esta ciudad. Así, una biblioteca va mucho más allá que otros espacios culturales, como los museos, por su propia naturaleza social, que no resulta incompatible con una presencia física atrayente. Se une así una más profunda e inmediata utilidad social con el efecto de creación de un hito urbano e incluso turístico; de un símbolo.

También el reciente estudio sobre el impacto de las bibliotecas en la sociedad que se realizó en Navarra (Hernández, 2016), en el marco de los trabajos que el Consejo de Cooperación Bibliotecaria y FESABID están llevando a cabo sobre el tema, demuestra la importancia de los espacios físicos para el desarrollo tanto de los servicios tradicionales como de los que nos referimos arriba. Así, como resultado de una encuesta realizada complementariamente al cálculo del (retorno de la inversión) ROI de las bibliotecas, se indican entre sus papeles beneficiosos y reconocidos por la comunidad el proporcionar espacio para el estudio, ocio, reunión o conectividad. 


\begin{tabular}{lr}
\hline \multicolumn{2}{l}{ Población que está de acuerdo en que las bibliotecas... } \\
\hline $\begin{array}{l}\text { Son un soporte para la educación } \\
\text { de los niños y jóvenes }\end{array}$ & $98,0 \%$ \\
\hline Dan espacio a los jóvenes para estudiar & $96,1 \%$ \\
\hline $\begin{array}{l}\text { Posibilitan un tiempo de ocio y } \\
\text { entretenimiento de calidad }\end{array}$ & $93,4 \%$ \\
\hline $\begin{array}{l}\text { Facilitan la formación permanente } \\
\text { en todas las edades }\end{array}$ & $91,1 \%$ \\
\hline \begin{tabular}{l} 
Facilitan acceso a Internet para todos \\
\hline Apoyan el desarrollo temprano de los niños
\end{tabular} & $90,7 \%$ \\
\hline \begin{tabular}{l} 
Facilitan lugares de reunión \\
\hline $\begin{array}{l}\text { Facilitan el aprendizaje y uso de ordenadores } \\
\text { y otros equipos }\end{array}$
\end{tabular} & $\mathbf{8 0 , 3 \%}$ \\
\hline Ayudan a la gente a prepararse para conseguir nuevos empleos & $77,5 \%$ \\
\hline Ayudana aprender idiomas & $60,8 \%$ \\
\hline $\begin{array}{l}\text { Fomentan el hábito, el disfrute y las } \\
\text { competencias de lectura * }\end{array}$ & $97,1 \%$ \\
\hline *Base: usuarios de bibliotecas públicas & $52,0 \%$ \\
\hline
\end{tabular}

Figura 1. Fuente: (Hernández, 2016, p. 37).

\subsection{Contexto regional del análisis}

En lo que se refiere al contexto de Castilla-La Mancha, su política bibliotecaria se desarrolla, como en el resto de las comunidades autónomas, durante los últimos 30 años, desde la aprobación de la Constitución Española de 1978, la creación del estado de las autonomías y los decretos de transferencia de las competencias de cultura. A pesar de ser corta en el tiempo, se caracteriza por haber sido intensa y fructífera, ya que a principios de los años noventa, recién aprobada la citada ley de bibliotecas, la estructura bibliotecaria actual prácticamente no existía. Ha sido una de las comunidades que más ha avanzado, con la aprobación de dos leyes de bibliotecas (1989 y 2011), la creación y apertura de su Biblioteca Regional de Castilla-La Mancha, inexistente hasta 1996, y de la Red de Bibliotecas Públicas. En el año 2010 obtuvo los datos más elevados en cuanto a población servida, llegando a atender al 93,5\% de sus municipios y al $99,71 \%$ de su población.

\begin{tabular}{lccc|cccc}
\hline TOTALES & & & & \multicolumn{4}{|c}{ INDICADORES } \\
\hline $\begin{array}{c}\text { Bibliotecas } \\
\text { abiertas }\end{array}$ & $\frac{\mathbf{2 0 0 5}}{519}$ & $\frac{\mathbf{2 0 0 9}}{556}$ & $\frac{\mathbf{2 0 1 3}}{506}$ & $\begin{array}{l}\text { Habitantes } \\
\text { por } \\
\text { biblioteca }\end{array}$ & $\frac{\mathbf{2 0 0 5}}{3,651}$ & $\frac{\mathbf{2 0 0 9}}{3,715}$ & $\frac{\mathbf{2 0 1 3}}{4,092}$ \\
$\begin{array}{c}\text { Municipios } \\
\text { atendidos }\end{array}$ & 553 & 758 & 797 & $\%$ & $60.2 \%$ & $82.5 \%$ & $86.7 \%$ \\
$\begin{array}{c}\text { Población } \\
\text { atendida }\end{array}$ & 1.894 .667 & 2.065 .461 & 2.070 .833 & $\%$ & $98.8 \%$ & $99.2 \%$ & $98.6 \%$ \\
\hline
\end{tabular}

Tabla I. Datos generales de las bibliotecas públicas de Castilla-La Mancha. 
El corpus legislativo bibliotecario de esta región se fundamenta principalmente en:

- La Ley 3/2011, de 24 de febrero, de la Lectura y de las Bibliotecas de Castilla-La Mancha.

- El Decreto 32/2013, de 13/06/2013, de organización y funcionamiento del Consejo de la Lectura y Bibliotecas de Castilla-La Mancha y del Consejo Asesor de la Red de Bibliotecas Públicas de Castilla-La Mancha.

- El Decreto 33/2013, de 13 de junio, de funcionamiento de la Comisión Técnica y de las Comisiones Técnicas Provinciales de la Red de Bibliotecas Públicas de Castilla-La Mancha.

Según estas normativas y la Ley 7/1985 Reguladora de las Bases del Régimen Local, las bibliotecas públicas municipales son servicios de competencia local, aunque la Ley 3/2011 dota además a las administraciones provinciales y regionales de Castilla-La Mancha de diferentes responsabilidades para impulsar la prestación de este servicio. Tiene una concepción mucho más ambiciosa que la normativa nacional al disponer que todos los municipios de la región mayores de 1.000 habitantes deberán disponer de biblioteca de titularidad pública y uso general, dándose el servicio en el resto (hasta 300 habitantes) mediante bibliotecas móviles. Además, la ley obliga a implantar una red en los municipios con más de 20.000 habitantes, aunque esto no se cumple en el caso de Guadalajara, que amparándose en la presencia de la Biblioteca Pública del Estado no tiene servicio bibliotecario municipal. Una excepción en una región que puede "presumir" de que solo hay 12 municipios con una población entre 2.000 y 750 habitantes que no tengan biblioteca.

Aunque las bibliotecas públicas, dentro de las políticas culturales locales y regionales, son servicios muy implantados en los pueblos y abarcan a casi la totalidad de la población regional en Castilla-La Mancha, los ayuntamientos las gestionan en la mayoría de los casos sin planificación y sin modelo. Y ello se refleja en las propuestas electorales de los partidos, que como veremos son pobres e inconexas al no existir planes estratégicos sobre este servicio en la mayoría de los ayuntamientos, y por tanto carecen de unas pautas o líneas a seguir. A pesar de los grandes avances bibliotecarios de las tres últimas décadas, impulsados sobre todo por las políticas del gobierno regional (la Junta de Comunidades de Castilla-La Mancha, la realidad es que a nivel municipal no han existido políticas bibliotecarias planificadas, sólo un seguimiento de las dinámicas generadas por la Junta.

Dentro de las políticas bibliotecarias desarrolladas en la región, la cuestión de los edificios estuvo presente a veces con la intervención estatal (caso de la construcción de las nuevas sedes de las Bibliotecas Públicas del Estado de Ciudad Real o Guadalajara, o la reforma de la de Cuenca) o autonómica, siendo los hitos en este segundo caso la inauguración de la Biblioteca Regional en Toledo, y el desarrollo de políticas de ayuda para la construcción y reforma de bibliotecas municipales, enmarcadas en el Plan de Desarrollo Bibliotecario 2003-2006, que tuvo como tercer objetivo:

3. Impulsar un programa de construcción, reformas y equipamiento de infraestructuras bibliotecarias adecuado a las necesidades de la biblioteca del siglo XXI. La biblioteca pública, con edificios modernos y bien dotados, debe ser el lugar de encuentro y de acceso a la cultura, la información, la educación y el uso responsable del tiempo libre para la comunidad local. Unas instalaciones adecuadas permitirán la mayor rentabilidad de sus colecciones y se convertirán en el mejor estandarte de la red de bibliotecas públicas.

Esto solo se concretó en una serie de medidas vinculadas con la construcción o reforma de las Bibliotecas Públicas del Estado y en el propósito de "publicación de una orden de la consejería estableciendo las características que han de tener los edificios, servicios y colecciones de las bibliotecas públicas".

Tras los años de crisis vemos que se ha sufrido un retroceso y queda mucho por hacer: aprobar el mapa de bibliotecas, firmar convenios con los ayuntamientos, seguir desarrollando la actual ley y, sobre todo, recuperar la financiación recortada en los cinco años anteriores. Ha habido una minoración muy importante de la financiación de las bibliotecas públicas por parte de todas las administraciones implicadas (tanto ayuntamientos como la Junta, el Ministerio de Educación, Cultura y Deportes, y las Diputaciones), bajando de forma drástica los presupuestos en adquisiciones, personal, infraestructura, edificios y mobiliario. Ello ha afectado a la situación bibliotecaria regional de Castilla-La Mancha, abocando al cierre de numerosas bibliotecas municipales, al despido de profesionales y al descenso de un $80 \%$ del gasto para adquisiciones y del $92 \%$ particularmente para inversiones, es decir, para infraestructuras e instalaciones. Si de 2005 a 2009 el gasto destinado a las inversiones había crecido un $74 \%$, en 2013 se había reducido de 8.199 .572 a sólo 664.736 euros: de 3.93 a 0.31 euros por habitante.

\begin{tabular}{lrr}
\hline Año & $\mathbf{2 0 0 9}$ & \multicolumn{1}{c}{$\mathbf{2 0 1 3}$} \\
\hline Total & $8.199 .572 €$ & $664.736 €$ \\
Gasto por habitante & $3,93 €$ & $0,31 €$ \\
\hline
\end{tabular}

Tabla II. Gasto en inversiones en Castilla-La Mancha. 


\section{RESULTADOS}

En la revisión de los programas electorales se extrajeron propuestas de muy diversos temas además de las centradas en edificios e instalaciones. Entre ellas cabe mencionar las relativas a la implantación de nuevos servicios, incremento de inversiones, adquisición o renovación del mobiliario, cuestiones de personal, ampliación de colecciones, promoción de la lectura, horarios de apertura, equipamientos informáticos, internet, fomento de la biblioteca, extensión cultural, mejoras del préstamo, formación de usuarios, apoyo a la biblioteca escolar o implantación del Consejo Asesor de la Biblioteca.

Entre las propuestas específicas sobre edificios se han incluido las concernientes a las bibliotecas de nueva creación en aquellos municipios donde no las había; las que demandaban bibliotecas sucursales para aquellos pueblos que plantean la creación o ampliación de su red municipal; las propuestas relacionadas con la construcción de un nuevo edificio para bibliotecas ya existentes; la ampliación o mejora de instalaciones; y las propuestas sobre mobiliario, por su evidente vinculación.

En las siguientes tablas (III, IV y V) se comparan las propuestas recogidas en los programas electorales municipales de los diferentes partidos políticos que han obtenido mayor número de votos en 2007 y 2015 ; mientras que en la Tabla VI se establece una comparación entre los totales de las propuestas de 2007 y de 2015 referidos a edificios.

\begin{tabular}{|c|c|c|c|c|c|c|}
\hline PROPUESTAS ELECTORALES & \multicolumn{2}{|r|}{ PSOE } & $\mathbf{P P}$ & IU & OTROS & Total \\
\hline EDIFICIO & \multicolumn{2}{|r|}{78} & 10 & 3 & 5 & 96 \\
\hline Biblioteca de nueva creación & \multicolumn{2}{|r|}{29} & 3 & & 1 & 33 \\
\hline Nuevo -Edificio & \multicolumn{2}{|r|}{28} & 4 & 3 & 3 & 38 \\
\hline Edificio-Ampliación & \multicolumn{2}{|r|}{12} & 2 & & & 14 \\
\hline Edificio-Mejoras & \multicolumn{2}{|r|}{9} & & & 1 & 10 \\
\hline RBM-Ampliación & & 1 & & & 1 \\
\hline MOBILIARIO & \multicolumn{2}{|r|}{10} & 1 & & & 11 \\
\hline \multicolumn{7}{|c|}{ Tabla III. Análisis comparativo de propuestas entre partidos a nivel municipal (2007). } \\
\hline $\begin{array}{l}\text { PROPUESTAS } \\
\text { ELECTORALES }\end{array}$ & $\mathbf{P P}$ & PSOE & Cs & IU & OTROS & Total \\
\hline EDIFICIO & 13 & 22 & 1 & 7 & 8 & 51 \\
\hline Biblioteca de nueva creación & & 3 & & & 1 & 4 \\
\hline Biblioteca-Sucursal & & 3 & & 1 & 2 & 6 \\
\hline Nuevo-Edificio & 3 & 4 & & 4 & 2 & 13 \\
\hline Edificio-Ampliación & 4 & 3 & & 1 & & 8 \\
\hline Edificio-Mejoras & 6 & 9 & 1 & 1 & 3 & 20 \\
\hline MOBILIARIO & 3 & 0 & 0 & 0 & 0 & 3 \\
\hline
\end{tabular}

Tabla IV. Análisis comparativo de propuestas entre partidos a nivel municipal (2015).

\begin{tabular}{lrrrrrr}
\hline $\begin{array}{c}\text { PROPUESTAS } \\
\text { ELECTORALES }\end{array}$ & PP & PSOE & Cs & IU & Otro & Total \\
\hline EDIFICIO & 12,87 & 13,75 & 16,6 & 16,6 & 11,59 & 14,31 \\
MOBILIARIO & 2,97 & 0,00 & 0,00 & 0,00 & 0,00 & 0,59 \\
\hline
\end{tabular}

Tabla V. Propuestas porcentuales por partidos políticos a nivel municipal en 2015. 


\begin{tabular}{|c|c|c|}
\hline PROPUESTAS ELECTORALES & 2007 & 2015 \\
\hline EDIFICIO & 96 & 51 \\
\hline Biblioteca de nueva creación & 33 & 4 \\
\hline Biblioteca sucursal & 1 & 6 \\
\hline Nuevo-Edificio & 38 & 13 \\
\hline Edificio-Ampliación & 14 & 8 \\
\hline Edificio-Mejoras & 10 & 20 \\
\hline MANTENIMIENTO DEL SERVICIO & 0 & 24 \\
\hline SERVICIOS & 15 & 41 \\
\hline CATÁLOGO COLECTIVO CLM & & 6 \\
\hline BIBLIOTECA ESCOLAR & 2 & 1 \\
\hline INVERSIONES & 3 & 7 \\
\hline MOBILIARIO & 11 & 3 \\
\hline Puestos de lectura-Ampliación & & 0 \\
\hline Mobiliario & & 2 \\
\hline Detector de hurtos & & 1 \\
\hline PERSONAL & 8 & 4 \\
\hline COLECCIONES & 37 & 45 \\
\hline LECTURA & 45 & 59 \\
\hline HORARIO & 26 & 52 \\
\hline SOFTWARE Y HARDWARE BCA. & 4 & 7 \\
\hline WEB BIBLIOTECA & 1 & 1 \\
\hline INTERNET & 28 & 21 \\
\hline FOMENTAR USO BIBLIOTECA & 23 & 11 \\
\hline EXTENSIÓN BIBLIOTECARIA & 6 & \\
\hline EXTENSIÓN CULTURAL & 22 & 29 \\
\hline PRÉSTAMO & 8 & 5 \\
\hline FORMACIÓN DE USUARIOS & 3 & 3 \\
\hline CONSEJO DE LA BIBLIOTECA & 1 & 1 \\
\hline ASOC. AMIGOS BIBLIOTECA & & 1 \\
\hline TOTAL & 339 & 372 \\
\hline
\end{tabular}

Tabla VI. Comparación de propuestas referidas a edificio entre 2007 y 2015.

En las elecciones de 27 de mayo de 2007 las cinco propuestas más presentes en los programas electorales a nivel municipal fueron edificios $(28,32 \%)$, lectura $(13,27 \%)$, fondos $(10,91 \%)$, internet $(8,26 \%)$ y horario $(7,67 \%)$. Transcurridos ocho años, las cinco propuestas más destacadas en los programas de las elecciones municipales de 2015 han pasado a ser: la promoción de la lectura $(15,86 \%)$, el horario $(13,97 \%)$, los edificios $(13,7 \%)$, colecciones $(12,09 \%)$ y servicios $(11,02 \%)$.

Entre los asuntos por los que han mostrado mayor interés los partidos políticos en estas elecciones municipales en 2015 vemos que los edificios aparecen con 51 propuestas, entre las que podemos mencionar las relacionadas con la creación de nuevas bibliotecas en aquellos municipios donde todavía no hay, que son un total de 4, y las relacionadas 
con la construcción de nuevos edificios, debido principalmente a instalaciones inadecuadas, insuficientes u obsoletas, que han sido 13 propuestas. Las destinadas a la ampliación de redes bibliotecarias con la creación de bibliotecas sucursales, con 6 propuestas y por último y más numerosas las destinadas a la ampliación y mejora de instalaciones, 8 y 20 respectivamente.

Asumiendo que para que exista una biblioteca debe haber una instalación, o más bien que la propuesta de creación de una biblioteca municipal se refiere sobre todo a las instalaciones, se puede indicar que, de los dos municipios mayores de 1.000 habitantes que no tienen biblioteca pública municipal según prescribe la ley, en Guadalajara sólo el PSOE ha propuesto en 2015 la creación de la misma, aunque ya lo hizo sin resultado práctico en 2007, año desde el que gobierna el PP en la ciudad. Y en El Casar de Escalona hasta ahora ningún partido lo ha propuesto. A su vez, de los 27 municipios donde se han recogido programas electorales con una población menor de 1.000 habitantes (que por tanto no tienen obligación legal de tenerla), 24 prestan ya este servicio, y en 17 de ellos se han realizado propuestas electorales por algún partido.

Así, de los 193 municipios de los que hemos obtenido programas electorales, sólo 6 de ellos no tienen todavía biblioteca pública, pero 3 de estos municipios no tienen obligación legal. En uno de los casos, Velada, se trataría de una reapertura.

Dos aspectos no directamente relacionados con la infraestructura, pero sí con su utilización, merecen ser destacados. Por una parte, en 2015 aparece claramente la preocupación por la permanencia misma del servicio, con 24 casos. Por otra, el interés por la amplitud de los horarios de apertura se dispara, duplicándose las referencias al tema, de 26 a 52.

En lo que se refiere al contenido de las propuestas, y aun existiendo numerosas relativas a la ampliación y mejora de las instalaciones, éstas se siguen refiriendo a aspectos convencionales de la biblioteca: ganar espacio para puestos de lectura y colecciones, mejoras de accesibilidad o cuestiones básicas de confort como instalar climatización.

Aunque el objeto de estudio de este trabajo sean los programas electorales, enriquece de forma indudable los resultados el conocimiento del grado de cumplimiento de las promesas electorales realizadas. De esta forma, en relación con la materialización de nuevos edificios e instalaciones en Castilla-La Mancha, y de acuerdo con los datos del Servicio Regional de Bibliotecas, hemos comprobado que entre 2007, tras las elecciones, y 2011 hubo 46 municipios que abrieron o construyeron nuevas bibliotecas o las trasladaron a nuevos espacios polivalentes.

Desafortunadamente, sólo se pudieron obtener programas del partido que resultó gobernante en 13 de ellos, lo que limita bastante la muestra. Así, ésta representa el $28 \%$ del total de municipios que llevaron a cabo alguna acción en materia de edificios. Entre ellos, el $61 \%$ de los partidos habían contemplado las propuestas en materia de edificios bibliotecarios que durante la legislatura 2007-2011 ejecutaron. Ello supone que el 39\% de las acciones en materia de edificios bibliotecarios no venían contempladas en sus programas electorales.

\section{DISCUSIÓN}

En 2015, las propuestas electorales a nivel municipal de todos los partidos han girado principalmente en torno a la promoción de la lectura y el horario de apertura, enfocándose casi siempre hacia la ampliación del mismo. Ello nos dice que hay una honda preocupación por la situación en la que se encuentra el servicio directo al ciudadano: de nada sirve la presencia de una infraestructura bibliotecaria si no se le dota de un servicio, uno de cuyos mínimos exigibles es un horario amplio, lo que a su vez requiere la presencia de al menos un profesional bibliotecario. El efecto de la crisis sobre esta cuestión se manifiesta en que los partidos planteen la cuestión del horario.

Después, aunque son la mitad de las encontradas en 2007, tenemos las propuestas sobre cuestiones relativas a los edificios, principalmente enfocadas ahora a ampliación y mejora de sus instalaciones. La crisis económica ha reconducido lo que ocurría en las elecciones municipales de 2007, cuando la mayoría de las propuestas sobre los edificios eran más ambiciosas, proponiéndose la construcción de nuevos edificios y la creación de nuevas bibliotecas, que sumaban casi el 30\% del total de las medidas encontradas en los programas. Es evidente la causa: en 2007 los ayuntamientos tenían unos presupuestos en los que preveían grandes ingresos, y los partidos políticos plantean políticas de expansión de servicios y nuevas instalaciones. En 2015, todavía en crisis económica, los presupuestos son sumamente austeros, no se planifican nuevas inversiones y las propuestas van dirigidas a intentar mejorar o arreglar lo existente, que siempre tendrá un coste menor que la construcción de nuevos edificios. 
El cambio de tendencia sitúa las infraestructuras en tercer lugar en cuanto a número de propuestas, pero el edificio sigue siendo uno de los principales problemas de las bibliotecas públicas, y es de valorar positivamente que los partidos políticos se impliquen incluyendo medidas en los programas para su construcción o mejora. Esta posición de privilegio entre las propuestas bibliotecarias parece indicar que las administraciones locales son conscientes de que los equipamientos actuales no cumplen los requerimientos óptimos suficientes y es necesario invertir en la renovación de sus edificios.

Por otra parte, se comprueba que las actuaciones en materia de edificio, horarios, personal, colecciones, actividades y servicios son los aspectos más destacados en los programas de ambas elecciones. Esto denota una preocupación por los aspectos bibliotecarios más visibles y sensibles de cara a los ciudadanos, pero que también constituyen los puntos cruciales para el buen funcionamiento de una biblioteca. Si alguno de ellos falla, las bibliotecas no prestarán un servicio de calidad que satisfaga plenamente a los ciudadanos.

En un análisis general nos resulta destacable también, entrando en el detalle del contenido de las propuestas, que se sigan ocupando de aspectos muy básicos y tradicionales, sin reflejar las necesidades de actualización de la biblioteca pública para adaptarse a los retos que determina la sociedad digital, incluyendo las reformas necesarias en los espacios bibliotecarios, quedándose anclados en modelos bibliotecarios obsoletos. En lo positivo, es importante valorar el enorme esfuerzo que realizan los municipios pequeños, menores de 1.000 habitantes, que sin tener obligación legal de tener biblioteca proponen su creación como servicio básico para los ciudadanos.

Resulta difícil establecer una comparación general entre partidos debido a la diversidad de muestras, pero estas son suficientes para subrayar la gran diferencia de propuestas del PSOE con respecto al resto de partidos. Por ejemplo, y con un número de programas recogidos semejante, 78 propuestas del PSOE frente a 10 del PP en 2007 y 22 frente a 13 en 2015. En el caso de 2007, especialmente notorio, probablemente se percibe aún la influencia de la notable política de desarrollo bibliotecario realizada por el gobierno regional durante años.

Si realizamos un análisis partido a partido encontramos lo siguiente:

PP

Con respecto a 2007, donde destacaban las propuestas sobre edificios, en 2015 éstas han disminuido, no tanto porque se haya resuelto el problema de una de las medidas bibliotecarias que más coste económico puede suponer en una legislatura, sino que los ayuntamientos no tienen presupuestos para inversiones. Más bien al contrario, el que todavía sean numerosas demuestra la preocupación por las instalaciones y malas infraestructuras bibliotecarias que tienen las bibliotecas municipales de Castilla-La Mancha. El mobiliario está presente en menor medida que los edificios, pero también aumentan ligeramente las propuestas sobre esta cuestión en las elecciones municipales de 2015.

PSOE

En las elecciones municipales de 2007 el Partido Socialista realizó la mayoría de sus propuestas en torno a la construcción de nuevos edificios y sobre la creación de nuevas bibliotecas, superando el 30\% del total de las mismas. Un porcentaje elevado teniendo en cuenta lo costoso de levantar nuevos edificios y poner en funcionamiento nuevos servicios. En 2015 el número de sus propuestas sobre este tema baja considerablemente (de 78 a 22), lo que se puede una vez más achacar a que en las elecciones de 2007 todavía no había comenzado la crisis económica que sigue vigente en las elecciones de 2015 y era comprensible que presentaran numerosas propuestas que suponían fuertes inversiones como son las infraestructuras. Con respecto a las propuestas sobre mobiliario, en 2015 ha pasado sorprendentemente a estar ausentes de los programas del Partido Socialista.

IU

Las infraestructuras bibliotecarias han sido unas de las mayores inquietudes de Izquierda Unida en ambas elecciones. En 2007 con un 30\%, y en 2015 con un 16,67\% de sus propuestas. De aquí se deduce su interés por la situación de muchos edificios bibliotecarios y de las carencias y necesidades que presentan, siendo conscientes de que es uno de los aspectos más negativos de las bibliotecas y que más pueden interesar a sus posibles votantes.

OTROS

También para los partidos minoritarios los espacios de biblioteca han supuesto una de las mayores preocupaciones en ambas elecciones, pero con una fortísima reducción: en 2007 con un 41,67\% y en 2015 con un $12,33 \%$. Observamos no obstante el interés por la situación de las infraestructuras bibliotecarios y su priorización en la agenda al incluirlas en sus programas.

Faltando los datos del incumplimiento de promesas de ejecución de bibliotecas entre los partidos que gobernaron, no se deben extraer conclusiones de la diferencia entre el número de propuestas, 72 si excluimos las ampliaciones y reformas, y las 46 que finalmente se llevaron a cabo. No obstante, sí nos permite intuir cierto grado de incumplimiento, probablemente vinculado a la caída de los presupuestos públicos y de las ayudas regionales en esta 
época. Consideramos que hay una correspondencia entre la inclusión de propuestas y su realización efectiva, pero también hemos constatado que el $39 \%$ de las acciones se realizaron en municipios en los que los partidos presentados no las habían incluido en los programas. Esto sería consecuencia de que hay otros factores generales de oportunidad o de política pública que influyen en el desarrollo del servicio bibliotecario. Estar en los programas es positivo, pero no el único factor para la adopción de medidas sobre el servicio bibliotecario.

\section{CONCLUSIONES}

Del estudio y discusión de los resultados puede extraerse que, si bien las bibliotecas siguen despertando interés en los partidos y, por tanto, en la sociedad, éstas son vistas aún desde un punto de vista muy tradicional. No se percibe la imagen de una biblioteca pública acorde con las actuales tendencias sobre biblioteca social, biblioteca ágora, biblioteca formadora, espacios de creación, etc. Todo ello exige de una renovación y reorientación de las infraestructuras y los espacios que no aparece en las propuestas estudiadas.

Por otra parte, en una época de claras dificultades presupuestarias, las propuestas electorales reflejan la necesidad del mantenimiento de unos servicios y prestaciones básicas, sin preocuparse por el desarrollo de las mismas ni, por tanto, de una significativa modernización. En las actuales circunstancias resulta muy complicado que las bibliotecas de Castilla-La Mancha adapten sus instalaciones y servicios a la nueva realidad, cuando en muchos casos está en peligro su propia supervivencia debido a la falta de recursos.

Las bibliotecas aparecen en los programas electorales, pero resulta discutible que resulten determinantes para la obtención del voto. La reducción de las propuestas de construcción y grandes reformas de centros indican que las bibliotecas no son prioritarias para la sociedad. Significativamente, en una época de crisis los partidos orientan las propuestas que suponen mayor gasto hacia otros temas.

Refrendando lo anterior, la aparición de la propuesta de construcción o apertura de una nueva biblioteca en los programas electorales no implica su obligado cumplimiento, sino que ésta parece vincularse más a la aparición de la oportunidad durante la legislatura.

Debido a todo ello, se hace necesario que desde el ámbito bibliotecario se profundice en la difusión del modelo actual de biblioteca en la sociedad y, en especial, en el ámbito político. También, el bibliotecario debe conocer las herramientas que tiene a su disposición para la justificación de su servicio, como los estudios sobre el retorno de la inversión (ROI) de las bibliotecas.

Todo ello requiere por parte del bibliotecario de un conocimiento profundo del funcionamiento de los sistemas políticos y de los mecanismos de elaboración de la agenda. También de su preparación en técnicas de negociación y la mejora de sus conocimientos de marketing; tanto para la promoción y difusión del servicio bibliotecario como para la mejora del mismo, aportando nuevos argumentos a los gestores y electos.

Si la influencia lobista y la presentación de soluciones a los problemas son factores de la conformación de la agenda, los bibliotecarios deben participar de ambas. Para ello, resulta fundamental la actuación coordinada, no sólo de forma individual, sino como grupo. Así, se precisa de organizaciones bibliotecarias verdaderamente sólidas e influyentes, en las que el camino a la convergencia entre las mismas lleve a una voz única y más fuerte.

\section{BIBLIOGRAFÍA}

BALLART, X. y RAMIÓ MATAS, C. Formulación de las políticas públicas. En Ciencia de la administración. Valencia: Tirant lo Blanch, 2000, p. 504-518.

BOUGHEY, A. y COOPER, M. Public libraries: political vision versus public demand? Aslib Proceedings [En línea], 2010, vol. 62, no 2, p. 175-201. Disponible en: <http://dx.doi.org/10.1108/00012531011034982>.

DESRICHARD, Y. (coord.). Politique(s) et bibliothèques: dossier. Bulletin des Biliothèques de France [En línea], 2011, vol. 56, n 2. Disponible en: <http://bbf.enssib.fr/sommaire/2011/2>. [Consulta: 12 de septiembre de 2016].

EVJEN, S. The image of an institution: Politicians and the urban library project. Library \& Information Science Research [En línea], 2015, vol. 37, p. 28-35. Disponible en: <http://dx.doi.org/10.1016/j.lisr.2014.09.004>.

GALLO-LEÓN, J.P. et al. Prospectiva 2020. Las diez áreas que más van a cambiar en nuestras bibliotecas en los próximos años [En línea]. Madrid: Consejo de Cooperación Bibliotecaria, 2013. Disponible en: $<$ http://www.mecd.gob.es/cultura-mecd/dms/mecd/cultura-mecd/areas-cultura/bibliotecas/mc/consejocb/gruposde-trabajo/21/estudio-prospectiva-2020.pdf>. [Consulta: 28 de septiembre de 2016].

GARCEAU, O. The public library in the political process. New York: Columbia University Press, 1949. 
GAZO, D. City councillors and the mission of public libraries. New Library World [En línea], 2011, vol. 112, n 1/2, p. 52-66. Disponible en: <http://dx.doi.org/10.1108/03074801111100454>.

GIL SOLÉS, D. La biblioteca líquida. Bauen: biblioteques i arquitectura [En línea], 2012. Disponible en: $<$ http://www.bauenblog.info/2012/12/19/la-biblioteca-liquida/>. [Consulta: 28 de septiembre de 2016].

HERNÁNDEZ-SÁNCHEZ, H. El valor de las bibliotecas. Estudio de Impacto Socioeconómico de las Bibliotecas de Navarra. Madrid: Consejo de Cooperación Bibliotecaria, 2016. Disponible en: $<$ http://www.elvalordelasbibliotecas.es/es/informe/>. [Consulta: 28 de septiembre de 2016].

JAEGER, P.T.; GORHAM, U.; BERTOT, J.C. y SARIN, L.C. Public Libraries, Public Policies, and Political Processes: Serving and Transforming Communities in Times of Economic and Political Constraint. Lanham, MD: Rowman \& Littlefield, 2014.

JAEGER, P.T.; BERTOT, J.C. y GORHAM, U. Wake Up the Nation: Public Libraries, Policy Making, and Political Discourse. Library Quarterly [En línea], 2013, vol. 83, $\mathrm{n}^{\mathrm{o}}$ 1, p. 61-72. Disponible en: $<$ http://dx.doi.org/10.1086/668582>.

JARAMILlO, O.; ÁLVAREZ ZAPATA, D. y MONCADA, P.D. Políticas públicas para bibliotecas públicas: una propuesta de soluciones locales a problemas globales. Investigación Bibliotecológica [En línea], 2005, vol. 19, $\mathrm{n}^{\circ}$ 39, p. 13-27. Disponible en: $<$ http://www.scielo.org.mx/scielo.php?script=sci_arttext\&pid=S0187358X2005000200002>. [Consulta: 8 de agosto de 2016].

JUNTA DE COMUNIDADES DE CASTILA-LA MANCHA. Plan Bibliotecas siglo XXI en Castilla-La Mancha: Plan de Desarrollo Bibliotecario (2003-2006). Toledo: Junta de Comunidades de Castila-La Mancha, 2003.

JUNTA DE COMUNIDADES DE CASTILA-LA MANCHA. Ley 3/2011, de 24 de febrero, de la Lectura y de las Bibliotecas de Castilla-La Mancha. Diario Oficial de Castilla-La Mancha [En línea], 8 de marzo de 2011, $\mathrm{n}^{\circ} 21$, p. 8992-9011. Disponible en: $<$ http://docm.jccm.es/portaldocm/descargarArchivo.do?ruta=2011/03/08/pdf/2011_3585.pdf\&tipo=rutaDocm>. [Consulta: 28 de septiembre de 2016].

LISON, B. et al. Research for Cult Committee: Public Libraries - Their New Role. Brussels: European Parliament, 2016

Disponible

en: $<$ http://www.europarl.europa.eu/RegData/etudes/STUD/2016/585882/IPOL_STU(2016)585882 EN.pdf>. [Consulta: 12 de septiembre de 2016].

MARÍN JIMÉNEZ, M.D. Política bibliotecaria y elecciones: propuestas electorales sobre bibliotecas públicas en las elecciones autonómicas y municipales de Castilla-La Mancha. [Tesis doctoral] [En línea]. Murcia: Universidad de Murcia, 2015. Disponible en: <http://hdl.handle.net/10803/33416>. [Consulta: 28 de septiembre de 2016].

MENESES TELLO, F. Bibliotecas y política: el paradigma político de la política. Anales de Documentación [En línea], 2013, vol. 16, no 2, p. 1-14. Disponible en: <http://dx.doi.org/10.6018/analesdoc.16.2.172471>.

MICHNIK, K. Public library managers' descriptions of political attention. Library Management [En línea], 2015, vol 36, $\mathrm{n}^{\circ}$ 8, p. 673-684. Disponible en: <http://search.proquest.com/docview/1753391758?accountid=17225>. [Consulta: 12 de septiembre de 2016]

NOGUERA, A. La elaboración democrática de los programas electorales: participación "versus" decisión. Temas para el debate, 2011, n 204, p. 38-40.

SÁNCHEZ SÁNCHEZ, J. Tiempo de elecciones, tiempo de bibliotecas. En Castilla-La Mancha [En línea], 16 de febrero de 2015. Disponible en: <http://www.encastillalamancha.es/noticia/53034/wwwencastillalamanchaes>. [Consulta: 22 de febrero de 2015].

SÁNCHEZ SÁNCHEZ, J. Bibliotecas públicas y partidos políticos: las políticas bibliotecarias en los programas electorales (1977-1993). Boletín de la ANABAD [En línea], 1994, vol. 44, no 2, p. 123-176. Disponible en: $<$ https:/dialnet.unirioja.es/descarga/articulo/50894.pdf $>$. [Consulta: 12 de septiembre de 2016].

SHAVIT, D. The Politics of Public Librarianship. New York: Greenwood, 1986.

SVEUM, T. y TVETER, F. Norwegian public libraries and governmental reports: visions and economic realities. New Library World. [En línea], 2012, vol. 113, $\mathrm{n}^{\mathrm{o}}$ 7/8, p. 351-363. Disponible en: $<$ http://dx.doi.org/10.1108/03074801211244977>.

TULEU, B. Trop loin, trop proche: les politiques et la bibliothèque. Bulletin des Bibliothèques de France [En línea], 2011, vol. 56, no 2, p. 14-17. Disponible en: <http://bbf.enssib.fr/consulter/bbf-2011-02-0014-002>. [Consulta: 12 de septiembre de 2016].

WAHNICH, S. À quoi sert une bibliothèque? Bulletin des Biliothèques de France [En línea], 2011, vol. 56, $\mathrm{n}^{\mathrm{o}} 2, \mathrm{p}$. 23-26. Disponible en: <http://bbf.enssib.fr/consulter/bbf-2011-02-0023-004>. [Consulta: 12 de septiembre de 2016].

WORPOLE, K. Contemporary library architecture: a planning and design guide. London y New York: Routledge, 2013. 